\title{
Different control applications on a vehicle using fuzzy logic control
}

\author{
NURKAN YAGIZ ${ }^{1}$, L EMIR SAKMAN ${ }^{1}$ and RAHMI GUCLU ${ }^{2}$ \\ ${ }^{1}$ Department of Mechanical Engineering, Istanbul University, Avcilar, Istanbul, \\ Turkey \\ ${ }^{2}$ Department of Mechanical Engineering, Yildiz Technical University, Besiktas, \\ Istanbul, Turkey \\ e-mail: guclu@yildiz.edu.tr
}

MS received 18 April 2006; revised 22 September 2007

\begin{abstract}
In this paper, the active suspension control of a vehicle model that has five degrees of freedom with a passenger seat using a fuzzy logic controller is studied. Three cases are taken into account as different control applications. In the first case, the vehicle model having passive suspensions with an active passenger seat is controlled. In the second case, active suspensions with passive passenger seat combination are controlled. In the third case, both the passenger seat and suspensions have active controllers. Vibrations of the passenger seat in the three cases due to road bump input are simulated. At the end of the study, the results are compared in order to select the combination that supplies the best ride comfort.
\end{abstract}

Keywords. Vehicle vibrations; active suspensions; fuzzy logic control; vehicle model.

\section{Introduction}

The suspension systems support the vehicle body, decrease the road surface induced vehicle vibrations, help to improve ride comfort and road holding. Springs and dampers are main components of the suspensions that are parallel to each other and placed between the vehicle body and axles. The optimum design of these elements is the main goal of the manufacturers. Two important factors of ride comfort and road holding which are conflicting each other have to be compromised. Hard springs result in better road holding whereas ride comfort gets worse. On the other hand, resonance of the linear and angular motions of the vehicle body is another source of the uncomfortable ride. Addition of active systems has the potential of improving ride comfort. A good vehicle suspension system has to reduce the sprung mass displacement together with acceleration and provide adequate suspension deflection to maintain tires on contact. This helps to improve ride comfort and vehicle maneuverability. Thus, the improvement of the active vehicle suspensions systems has attracted more interest and been the subject of the research and development in last decades. The reason is commercial as well as being scientific. The main aim of the commercial activity is the desire of the 
automotive manufacturers to improve the performance and quality of their products. On the other hand, researchers and control system designers have claimed that the active control of the vehicle suspension system is possible when the developments in actuators, sensors and electronics have been considered. In the last twenty years, many studies have been published on active and semi-active suspension systems. An optimal linear preview control on the active suspensions of a vehicle model was applied (Hac 1992). Hrovat (1993) surveyed applications of optimal control techniques for the design of active suspensions in one of his studies starting with a quarter model. The non-linear control of a quarter car active suspension was described (Alleyne \& Hedrick 1995). It was proposed an optimal preview type active suspension with feedback control based on easily measured relative displacements, velocities and accelerations (Thomson \& Pearce 1998). Sliding mode control on a full vehicle model was applied (Yagiz \& Yuksek 2001).

Because of continuously changing elevation of the road surface, classical approach of control algorithms degenerate the suspension gap preventing the suspensions and controllers from functioning and causes a very harsh ride. The aim of this study was to solve this problem with fuzzy logic controller. Fuzzy logic was first presented to the consideration of the academic society by Zadeh (1965) and became popular rapidly. Rao \& Prahlad (1997) suggested fuzzy logic based control for vehicle active suspension. The unsprung mass vibration on a quarter car model is controlled to reduce the related vertical acceleration. It was concerned with an active suspension system for motor coaches, using a skyhook damper and fuzzy logic control (Yoshimura et al 1997). The active control is assumed to be the sum of the skyhook damper and the fuzzy logic control. Again, Yoshimura et al (1999) presented an active suspension system for passenger cars, using linear and fuzzy-logic controls in their study. The model was described by a non-linear system with four degrees of freedom. The development of a composite controller for an automobile active suspension system was developed (Kuo \& Li 2000). Simulations were performed on a quarter car suspension model. A methodology was presented for the design of active car suspension systems. The aim of the study was to reduce the car body vertical acceleration to improve the passenger comfort and to avoid hitting suspension limits (D'Amato \& Viassolo 2000). The reactive component of motion control architecture for a car-like vehicle intended to move in dynamic partially known environments was presented (Fraichard \& Garnier 2001). Nizar et al (2002) designed a sliding mode neural network inference fuzzy logic controller to improve the ride and comfort. The ability of symbiotic evolution, as a generic methodology, to elicit a fuzzy rule-base of the Mamdani type was improved (Hyniova et al 2001; Jamei et al 2004). Sakman et al (2005) examined the performance of the fuzzy logic controlled active suspensions on a non-linear vehicle model having four degrees of freedom without causing any loss in suspension working limits. In this study, the ride comfort performances of the three control combinations are compared. First, only the unsprung mass is controlled through active suspensions. Second, only the passenger seat is controlled. Third, they are both controlled at the same time. The aim of this study is to decide the best control combination to improve the ride comfort without causing any degeneration in suspension working space using fuzzy logic control algorithm.

\section{The half vehicle model}

The half vehicle model is shown in figure 1. The fuzzy logic controllers have been placed between sprung and unsprung masses and between sprung mass and the driver seat. This model has five degrees of freedom which are the body bounce $x_{1}$, body pitch $x_{2}$, front wheel 


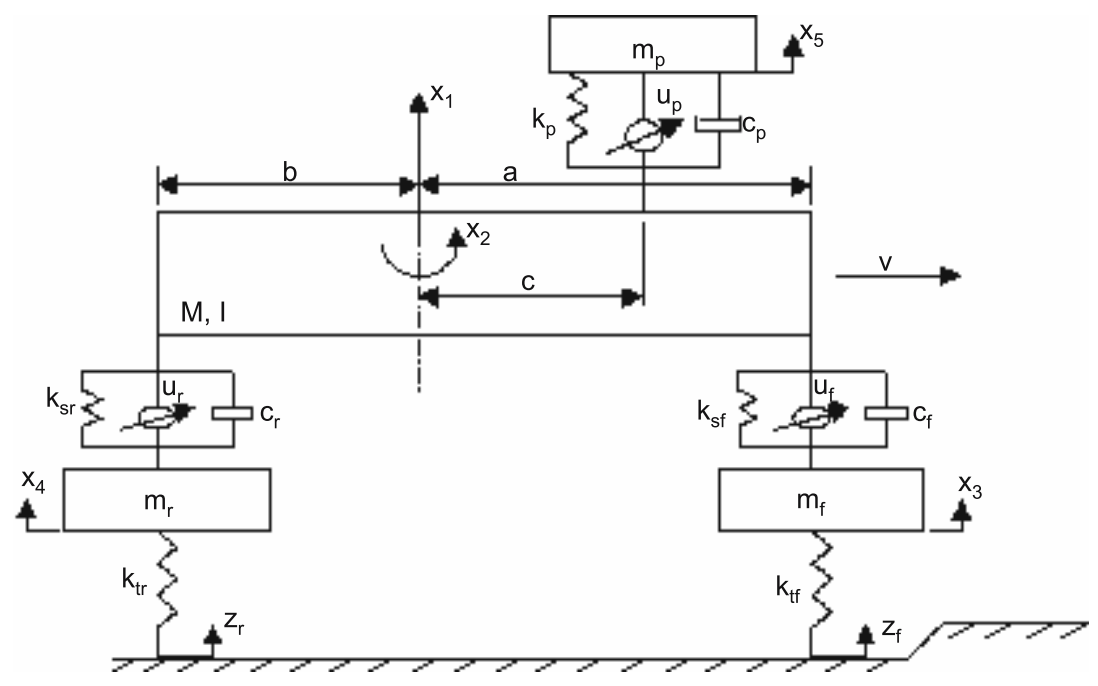

Figure 1. The vehicle model with five degrees of freedom.

hop $x_{3}$, rear wheel hop $x_{4}$ and passenger seat bounce $x_{5} . \mathbf{M}, I, m_{f}, m_{r}$ and $m_{p}$ are body mass, body inertia, front unsprung mass, rear unsprung mass, and passenger seat mass, respectively. $k_{s f}$ and $k_{s r}$ are front and rear suspension spring constants; $k_{t f}$ and $k_{t r}$ are the stiffness of front and rear wheels; $c_{f}$ and $c_{r}$ are front and rear suspension damping coefficients; $k_{p}$ and $c_{p}$ are the stiffness and damping coefficients of the arrangement under the passenger seat; $u_{f}, u_{r}$ and $u_{p}$ are control inputs to the front, rear and passenger seat of the vehicle; $z_{f}(t)$ and $z_{r}(t)$ are the front and rear wheel road surface inputs, respectively. Since the damping values of the wheels are very small and conventionally negligible, they are assumed to be zero. Vehicle parameters are given in the Appendix (A).

The general expression of the mathematical model is shown below:

$$
\dot{x}=f(x)+\mathbf{B} u
$$

where $\boldsymbol{x}=\left[x_{1} x_{2} x_{3} x_{4} x_{5} x_{6} x_{7} x_{8} x_{9} x_{10}\right]^{T}$ with states $x_{6}$ to $x_{10}$ are the derivatives of the first five, $f(x)$ is the vector of the equations of the system (Appendix B) excluding the control inputs which can be non-linear, $\mathbf{B}$ is the input matrix and $\boldsymbol{u}=\left[u_{f} u_{r} u_{p}\right]^{T}$ is the vector of the three control forces for the current model. The complete set of these equations and $B$ are given in the Appendix (C).

\section{The fuzzy logic controller}

Negative Medium, Negative Small, Zero, Positive Small and Positive Medium are used to represent the fuzzy value of the controller input and output as linguistic variables. In this study, their values are between 0 and 1 . Design of a fuzzy logic controller has following steps:

(1) Selecting suitable linguistic variables using the vehicle model parameters (fuzzification).

(2) To set-up suitable linguistic control rule base.

(3) To design a suitable control algorithm that bases on the measurable system variables. 
(4) Choosing a method for defuzzification. An interface to scale and map the linguistic control actions inferred to yield a non-fuzzy control input to the vehicle being controlled (defuzzification).

The fuzzy logic algorithm changes each input from the vehicle system parameters into the concern of fuzzy variable value using related fuzzy sets and suitable membership functions. Fuzzy sets and their suitable membership functions have to be defined for each output. The defuzzyfier is responsible for the translation of the fuzzy reasoning engine results into a crisp set of output values. A variety of methods could be used to perform defuzzyfication. In this study, the Mamdani method that returns the centroid of the output fuzzy region as the crisp output of the fuzzy interface system has been used (Yagiz et al 2002).

In traditional control logic of any type for active suspensions, the reference value for the vehicle body bounce and angular motions are assigned as zero. Assigning absolute zero value with respect to earth for the vehicle body bounce motion means compensating the change in the elevation of the vehicle by using the suspension working space. For example, if the road gains a certain elevation, the force actuator pulls the vehicle body down by losing suspension working space as much as the road irregularity. If the road loses any elevation, this time the suspension working space is forced to elongate to reach the zero displacement in vehicle body motion. Continuous changes in road surface elevation results in not functioning suspensions because of their working limits are exceeded. Therefore, the vehicle body suspends on the wheels having single degree of freedom and new resonance value of around $4-5 \mathrm{~Hz}$, which is uncomfortable for the human beings resulting in a harsh ride. To solve this problem, a fuzzy logic control method is designed which uses the vehicle body velocity and acceleration as multi input variables while the control force $(u)$ is the single output. The FAM table for the fuzzy controller is given in table 1. PM, NM, ZE, PS, NS, N and P mean Positive Medium, Negative Medium, Zero, Positive Small, Negative Small, Negative and Positive, respectively. ' $e$ ' represents the error of the related variables in this table.

\section{Comparison of control applications}

The road bump input is shown in figure 2 . The vehicle model in all cases travels over the speed bump at $70 \mathrm{~km} / \mathrm{h}$. The profile of the rear road input is a delayed version of the front input.

The simulation results for both active and passive controls have been presented in figures 3a-f for comparison. Three different control cases have been considered for the different active control device placements. In the case of only the control input $u_{p}$ being effective while control inputs $u_{f}$ and $u_{r}$ are zero, the vertical displacement of passenger seat is shown in figure 3a. Similarly, when the control inputs $u_{f}, u_{r}$ are effective and $u_{p}$ zero, the displacement of passenger seat is given in figure $3 \mathrm{~b}$. When all three of these control inputs $u_{f}, u_{r}$ and $u_{p}$ are applied, the results obtained are presented in figure $3 \mathrm{c}$. The controlled system results are given together with uncontrolled system results in all graphs. In figure $3 \mathrm{~d}$ through $3 \mathrm{f}$, the simulations are realized for vertical acceleration of the passenger seat. Since the acceleration values of the mass and inertia of the passengers are directly proportional with the force and moment values affecting them, it becomes important to check the acceleration performance of the controllers.

The improvement in the ride comfort by using active control is shown in figures $3 \mathrm{a}-\mathrm{f}$. The amplitudes of passenger seat bounce motion and vertical acceleration are decreased by using different control applications. In conventional methods, there stays a change in suspension 
Table 1. FAM table of the fuzzy controller.

\begin{tabular}{llllll}
\hline$e\left(\dot{x_{1}}-\dot{x_{f}}\right)$ & $e\left(\dot{x_{1}}\right)$ & \multicolumn{2}{l}{$e\left(\ddot{x_{1}}\right)$} & & $u$ \\
\hline PM & PM & ZE & P or N & ZE & PS \\
PS & PM & ZE & P or N & PS & PM \\
ZE & PM & ZE & P or N & PM & PB \\
NS & PM & ZE & P or N & PM & PB \\
NM & PM & ZE & P or N & PB & PVB \\
PM & PS & ZE & P or N & ZE & PS \\
PS & PS & ZE & P or N & PS & PM \\
ZE & PS & ZE & P or N & PS & PM \\
NS & PS & ZE & P or N & PM & PB \\
NM & PS & ZE & P or N & PM & PB \\
PM & ZE & ZE & P or N & NS & NM \\
PS & ZE & ZE & P or N & ZE & NS \\
ZE & ZE & ZE & P or N & ZE & ZE \\
NS & ZE & ZE & P or N & ZE & PS \\
NM & ZE & ZE & P or N & PS & PM \\
PM & NS & ZE & P or N & NM & NB \\
PS & NS & ZE & P or N & NM & NB \\
ZE & NS & ZE & P or N & NS & NM \\
NS & NS & ZE & P or N & NS & NM \\
NM & NS & ZE & P or N & ZE & NS \\
PM & NM & ZE & P or N & NB & NVB \\
PS & NM & ZE & P or N & NM & NB \\
ZE & NM & ZE & P or N & NM & NB \\
NS & NM & ZE & P or N & NS & NM \\
NM & NM & ZE & P or N & ZE & NS \\
\hline
\end{tabular}

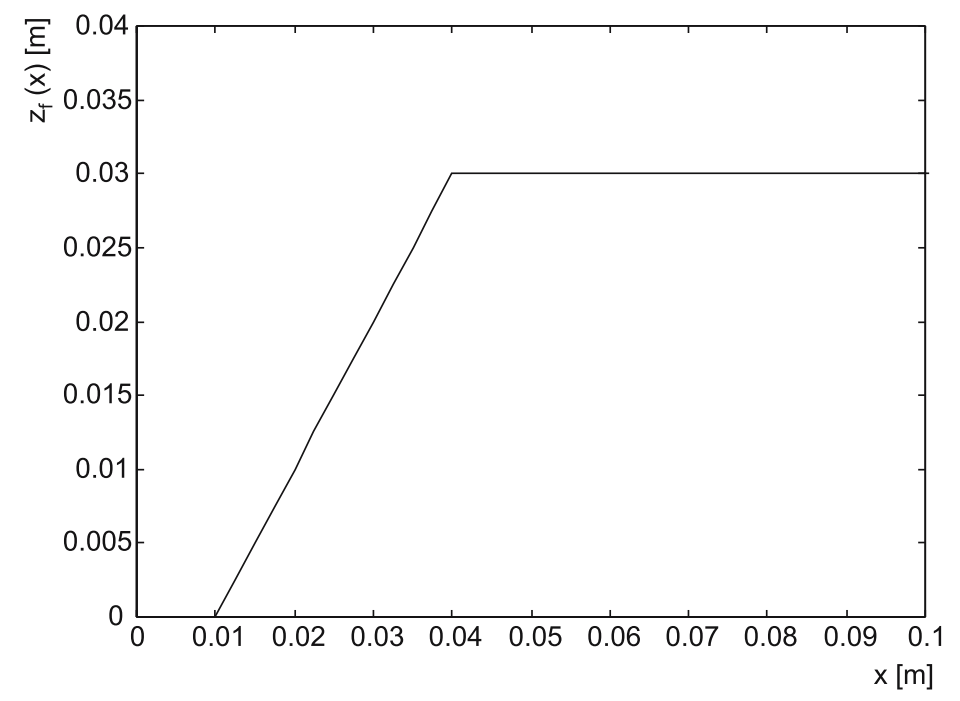

Figure 2. Road disturbance input. 

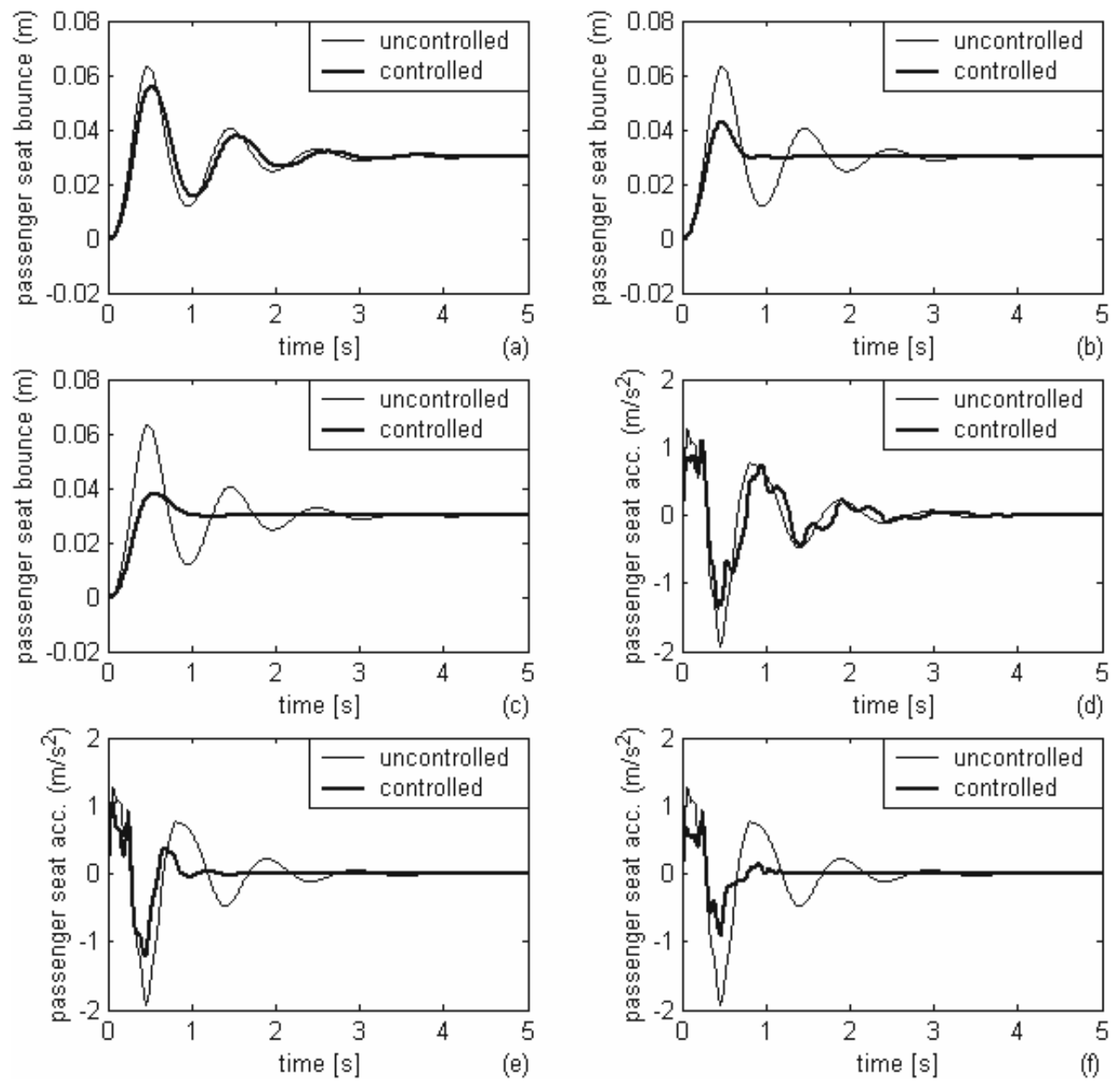

Figure 3. Vehicle passenger seat displacements and accelerations. (a) Only passenger seat controlled; (b) Only suspension controlled; (c) Fully controlled; (d) Only passenger seat controlled; (e) Only suspension controlled; (f) Fully controlled.

length, but in FLC proposed in this study, because the steady state position of the vehicle body mass displacement is the same as the road surface elevation, there will not be any loss in suspension working space length under control. In figures $4 \mathrm{a}, \mathrm{b}, \mathrm{c}$, the changes in controlled and uncontrolled suspension lengths are plotted. There is no permanent change in fuzzy logic controlled suspension length. In addition, as seen in this figure, the fuzzy logic controlled suspension reaches its original length faster and smoother than the uncontrolled one does. Figure 5a presents the time history of the control force when the controller exists only under the passenger seat. Control force inputs are also plotted in figures $5 \mathrm{~b}$ and $5 \mathrm{c}, \mathrm{d}$ for the other control alternatives. There is almost no change in the control forces for the second and third case and in the first case, the passenger seat controller force is relatively small when compared to the front and rear control forces. The maximum value of the front and rear control forces is about $800 \mathrm{~N}$. But the passenger seat controller applies as small as $100 \mathrm{~N}$. Thus a smaller and cheaper actuator will considerably increase the performance. 

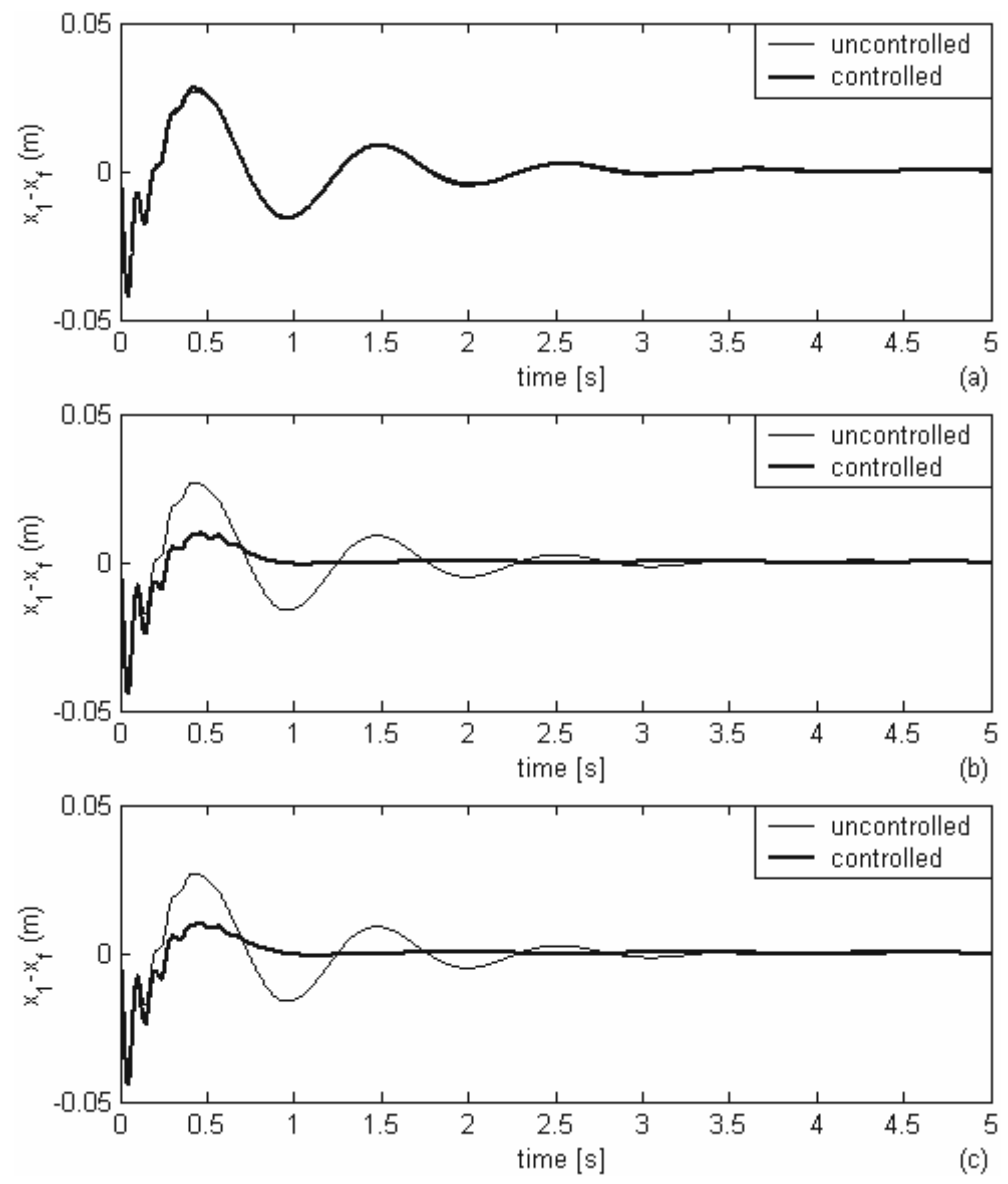

Figure 4. Gap responses for different control applications. (a) Only passenger seat controlled; (b) Only suspension controlled; (c) Fully controlled.

As seen in figures $6 \mathrm{a}$ and $6 \mathrm{~b}$, the frequency response of passenger seat displacement and acceleration for three control alternatives are presented together with the uncontrolled system response. There seem three resonance frequencies for the passive system. They belong to passenger seat, sprung mass (vehicle body) and unsprung masses. Actually, the vehicle model has five resonance frequencies. However, two of sprung mass and also two of unsprung masses overlap on each other.

If only the passenger seat is controlled, the resonance of the passenger is vanished and the number of resonances is decreased to two belonging to sprung and unsprung masses. If only the vehicle body is controlled, the number of resonances is decreased to two belonging to passenger seat and unsprung masses. When vehicle body and passenger seat are controlled at the same time, the amplitudes of resonance frequencies of almost all degrees of freedom decrease and the resonances of passenger seat and vehicle body vanish as a result of the successful performance of the fuzzy logic controller. 

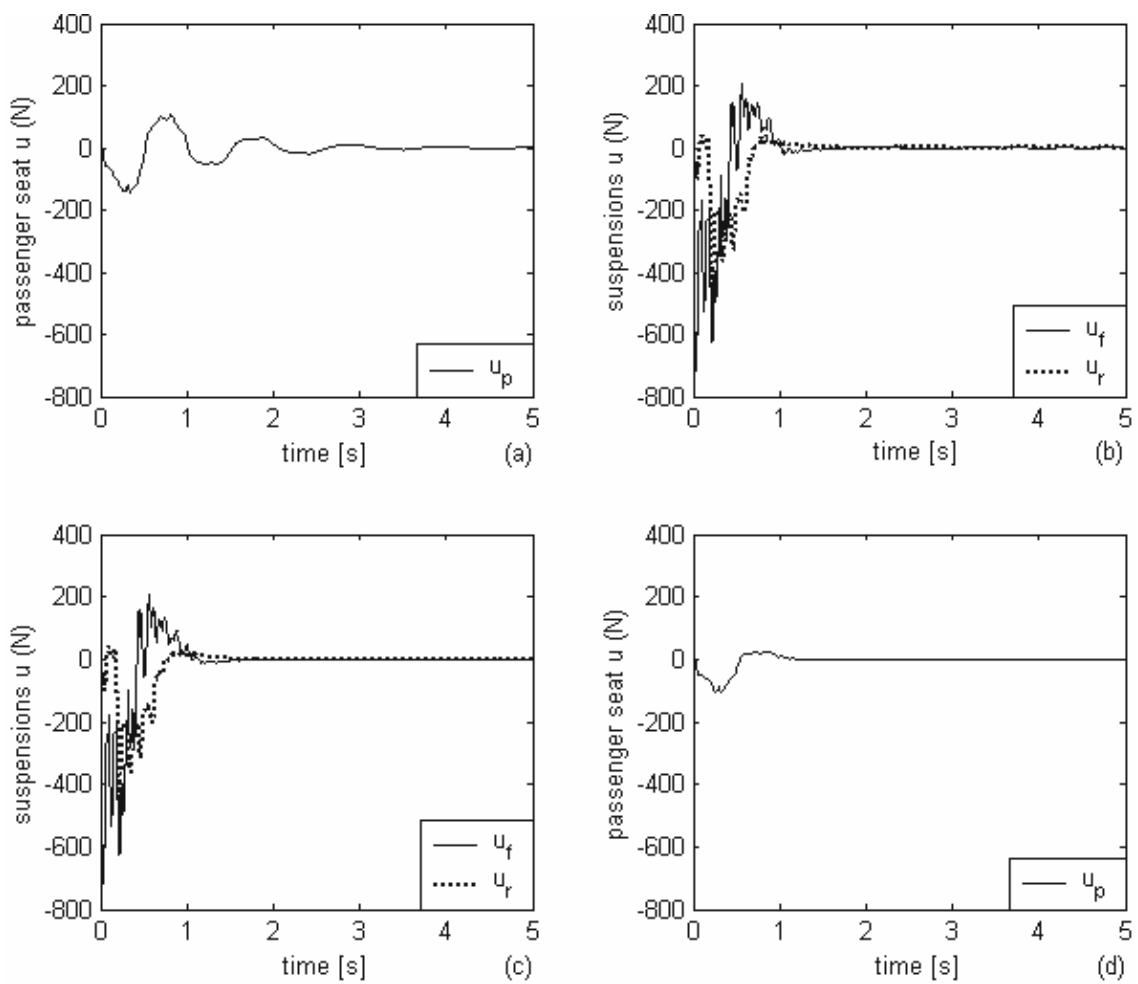

Figure 5. Control forces for the different control applications. (a) Only passenger seat controlled; (b) Only suspension controlled; (c) Fully controlled; (d) Fully controlled.
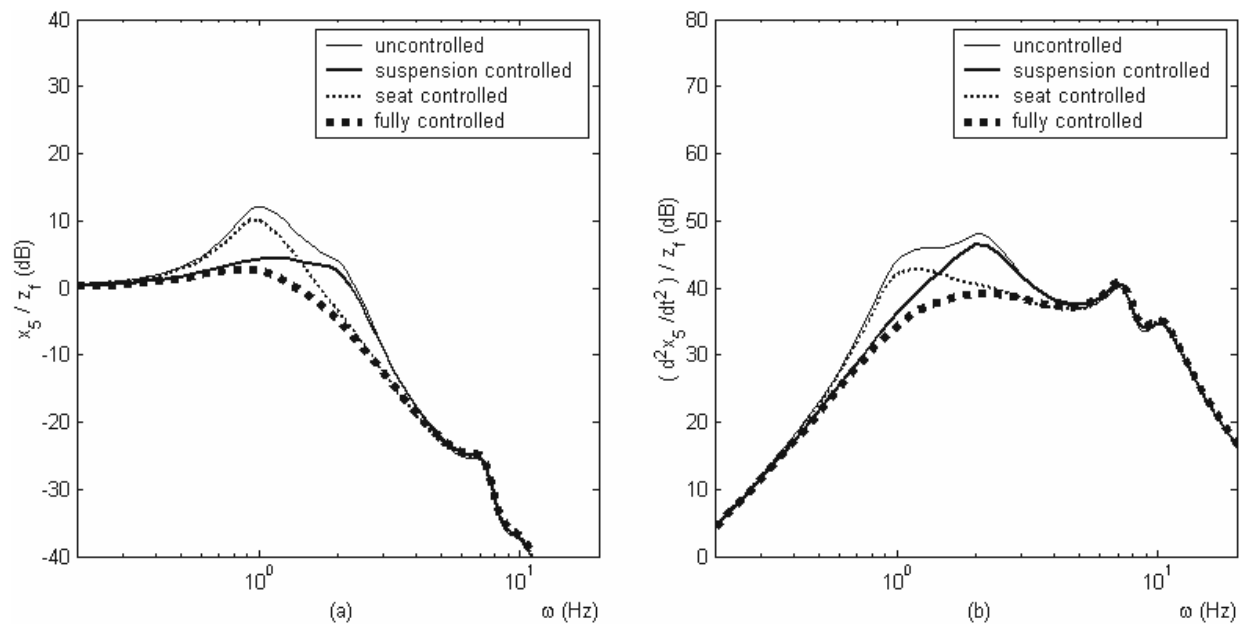

Figure 6. Frequency response of passenger seat displacement and acceleration. (a) Passenger seat displacements; (b) Passenger seat accelerations. 


\section{Conclusions}

In this paper, five degrees of freedom vehicle model having a passenger seat has been controlled using three different approaches. These are, at first, the vehicle model with passenger seat having a controller under it; secondly, with passive passenger seat but suspensions having controllers parallel to them; thirdly, with passenger seat having a controller under it together with suspensions having controllers parallel to them, respectively. The second case seems to be better than the first one while the third case is being the best. The results have been proved that, among three control strategies considered, using controllers under the vehicle body and passenger seat at the same time would provide the best ride comfort. Since adding a controller under the passenger seat together with the active suspensions improves ride comfort the most and does not cost much, the third control application is to be preferred.

\section{List of symbols}

$\begin{array}{ll}\text { B } & \text { control force matrix } \\ c_{f} & \text { front suspension damping } \\ c_{p} & \text { passenger seat damping } \\ c_{r} & \text { rear suspension damping } \\ \boldsymbol{f}(\boldsymbol{x}) & \text { vector of state equations without control inputs } \\ I & \text { body inertia } \\ k_{p} & \text { passenger seat stiffness } \\ k_{s f} & \text { front suspension stiffness } \\ k_{s r} & \text { rear suspension stiffness } \\ k_{t f} & \text { front wheel stiffness } \\ k_{t r} & \text { rear wheel stiffness } \\ \mathbf{M} & \text { body mass } \\ m_{f} & \text { front unsprung mass } \\ m_{p} & \text { passenger seat mass } \\ m_{r} & \text { rear unsprung mass } \\ \boldsymbol{u} & \text { vector of control inputs } \\ u_{f} & \text { front suspension control input } \\ u_{p} & \text { passenger seat control input } \\ u_{r} & \text { rear suspension control input } \\ \boldsymbol{x} & \text { vector of state variables } \\ \boldsymbol{x}_{\boldsymbol{r}} & \text { vector of state references } \\ x_{1} & \text { body bounce } \\ x_{2} & \text { body pitch } \\ x_{3} & \text { front wheel hop } \\ x_{4} & \text { rear wheel hop } \\ x_{5} & \text { passenger seat bounce } \\ z_{f}(t) & \text { front wheel road surface input } \\ z_{r}(t) & \text { rear wheel road surface input } \\ & \end{array}$




\section{Appendices}

\section{Appendix A}

Vehicle parameters

$$
\begin{array}{llll}
M=1000 \mathrm{~kg} & m_{p}=100 \mathrm{~kg} & k_{s f}=28000 \mathrm{~N} / \mathrm{m} & k_{t r}=400000 \mathrm{~N} / \mathrm{m} \\
I=1600 \mathrm{~kg} \mathrm{~m}{ }^{2} & c_{f}=2000 \mathrm{Ns} / \mathrm{m} & k_{s r}=32000 \mathrm{~N} / \mathrm{m} & a=1 \mathrm{~m} \\
m_{f}=100 \mathrm{~kg} & c_{r}=2000 \mathrm{Ns} / \mathrm{m} & k_{p}=16000 \mathrm{~N} / \mathrm{m} & b=1.2 \mathrm{~m} \\
m_{r}=200 \mathrm{~kg} & c_{p}=500 \mathrm{Ns} / \mathrm{m} & k_{t f}=400000 \mathrm{~N} / \mathrm{m} & c=0.5 \mathrm{~m}
\end{array}
$$

\section{Appendix B}

State equations excluding the control inputs

$$
\begin{aligned}
f_{1}(x)= & x_{6}, f_{2}(x)=x_{7}, f_{3}(x)=x_{8}, f_{4}(x)=x_{9}, f_{5}(x)=x_{10} \\
f_{6}(x)= & -1 / M\left\{\left(k_{s f}+k_{s r}+k_{p}\right) x_{1}+\left(a k_{s f}-b k_{s r}+c k_{p}\right) x_{2}\right. \\
& -k_{s f} x_{3}-k_{s r} x_{4}-k_{p} x_{5}+\left(c_{f}+c_{r}+c_{p}\right) x_{6} \\
& \left.+\left(a c_{f}-b c_{r}+c c_{p}\right) x_{7}-c_{f} x_{8}-c_{r} x_{9}-c_{p} x_{10}\right\} \\
f_{7}(x)= & -1 / I\left\{\left(a k_{s f}-b k_{s r}+c k_{p}\right) x_{1}+\left(a^{2} k_{s f}-b^{2} k_{s r}+c^{2} k_{p}\right) x_{2}\right. \\
& -a k_{s f} x_{3}+b k_{s r} x_{4}-c k_{p} x_{5}+\left(a c_{f}-b c_{r}+c c_{p}\right) x_{6} \\
& \left.+\left(a^{2} c_{f}+b^{2} c_{r}+c^{2} c_{p}\right) x_{7}-a c_{f} x_{8}+b c_{r} x_{9}+c c_{p} x_{10}\right\} \\
f_{8}(x)= & 1 / m_{f}\left\{k_{s f} x_{1}+a k_{s f} x_{2}-\left(k_{s f}+k_{t f}\right) x_{3}+c_{f} x_{6}+a c_{f} x_{7}\right. \\
& \left.-c_{f} x_{8}+k_{t f} z_{f}(t)\right\} \\
f_{9}(x)= & 1 / m_{r}\left\{k_{s r} x_{1}-b k_{s r} x_{2}-\left(k_{s r}+k_{t r}\right) x_{4}+c_{r} x_{6}-b c_{r} x_{7}\right. \\
& \left.-c_{r} x_{9}+k_{t r} z_{r}(t)\right\} \\
f_{10}(x)= & 1 / m_{p}\left\{k_{p} x_{1}+c k_{p} x_{2}-k_{p} x_{5}+c_{p} x_{6}+c c_{p} x_{7}-c_{p} x_{10}\right\}
\end{aligned}
$$

\section{Appendix C}

Input matrix for fully controlled case

$$
\mathbf{B}=\left[\begin{array}{cccccccccc}
0 & 0 & 0 & 0 & 0 & -\frac{1}{M} & -\frac{a}{I} & \frac{1}{m_{f}} & 0 & 0 \\
0 & 0 & 0 & 0 & 0 & -\frac{1}{M} & \frac{b}{I} & 0 & \frac{1}{m_{r}} & 0 \\
0 & 0 & 0 & 0 & 0 & \frac{1}{M} & \frac{c}{I} & 0 & 0 & -\frac{1}{m_{p}}
\end{array}\right]^{T}
$$




\section{References}

Alleyne A, Hedrick J K 1995 Non-linear adaptive control of active suspensions. IEEE Transactions on Control Systems Technology, 3(1): 95-101

D'Amato F J, Viassolo D E 2000 Fuzzy control for active suspensions. Mechatronics, 10: 897-920

Fraichard T, Garnier P 2001 Fuzzy control to drive car-like vehicles. Robotics and Autonomous Systems, 34: 1-22

Hac A 1992 Optimal linear preview control of active vehicle suspension. Vehicle System Dynamics, 21: $167-195$

Hrovat D 1993 Applications of optimal control to advanced automotive suspension design. Transactions of ASME, 115: 328-342

Hyniova K, Stribrsky A, Honcu J 2001 Fuzzy control of mechanical vibrating systems. Proceedings of International Carpathian Control Conference, Krakow, 393-398

Jamei M, Mahfouf M, Linkens D A 2004 Elicitation and fine-tuning of fuzzy control rules using symbiotic evolution. Fuzzy Sets and Systems, 147: 57-74

Kuo Y P, Li T H S 2000 A composite EP-based fuzzy controller for active suspension system. International J. Fuzzy Systems, 2(3): 183-190

Nizar H, Lahdhiri T, Joo D S, Weaver J, Faysal A 2002 Sliding mode neural network inference fuzzy logic control for active suspension systems. IEEE Transactions of Fuzzy Systems, 10(2): 234-246

Rao M V C, Prahlad V 1997 A tunable fuzzy logic controller for vehicle active suspension systems. Fuzzy Sets and Systems, 85: 11-21

Sakman L E, Guclu R, Yagiz N 2005 Fuzzy logic control of vehicle suspensions with dry friction non-linearity. Sādhana-Academy Proceedings in Engineering Sciences, 30(5): 649-659

Thomson A G, Pearce C E M 1998 Physically realizable feedback controls for a fully active preview suspension applied to a half-car model. Vehicle System Dynamics, 30: 17-35

Yagiz N, Yuksek I 2001 Sliding mode control of active suspensions for a full vehicle model. Int. J. Vehicle Design, 26(2/3): 264-276

Yagiz N, Guclu R, Yuksek I 2002 Fuzzy logic control of vehicle suspension systems for bumpy roads. The Journal of the Yildiz Technical University, 3: 75-82

Yoshimura T, Isari Y, Li Q, Hino J 1997 Active suspension of motor coaches using skyhook damper and fuzzy logic control. Control Engineering Practice, 5(2): 175-184

Yoshimura T, Nakaminami K, Kurimoto M, Hino J 1999 Active suspension of passenger cars using linear and fuzzy-logic controls. Control Engineering Practice, 7: 41-47

Zadeh L 1965 Fuzzy sets. Information and Control, 8: 338-353 